Hélène MOREL ${ }^{1}$

Éric NICOLINI ${ }^{2}$

\title{
Qualité et usages du bois de cinq espèces forestières adaptées à la plantation à vocation de bois d'œuvre et testées en Guyane française
}

Julie Bossu ${ }^{3}$

Lilian BLANC ${ }^{4}$

Jacques BEAUCHÊNE ${ }^{1}$

${ }^{1}$ Cirad, UMR EcoFoG

(AgroParisTech, Cirad, Inra, Université des Antilles, Université de Guyane)

97387 Kourou

France

${ }^{2}$ Cirad, UMR Amap

(Cirad, CNRS, Inra, IRD, Université

de Montpellier)

97387 Kourou

France

${ }^{3}$ CNRS, UMR ECoFoG

(AgroParisTech, Cirad, Inra, Université des Antilles, Université de Guyane) 97387 Kourou

France

${ }^{4}$ Cirad

UPR Forêts et Sociétés

Campus de Baillarguet

34398 Montpellier Cedex 5

France

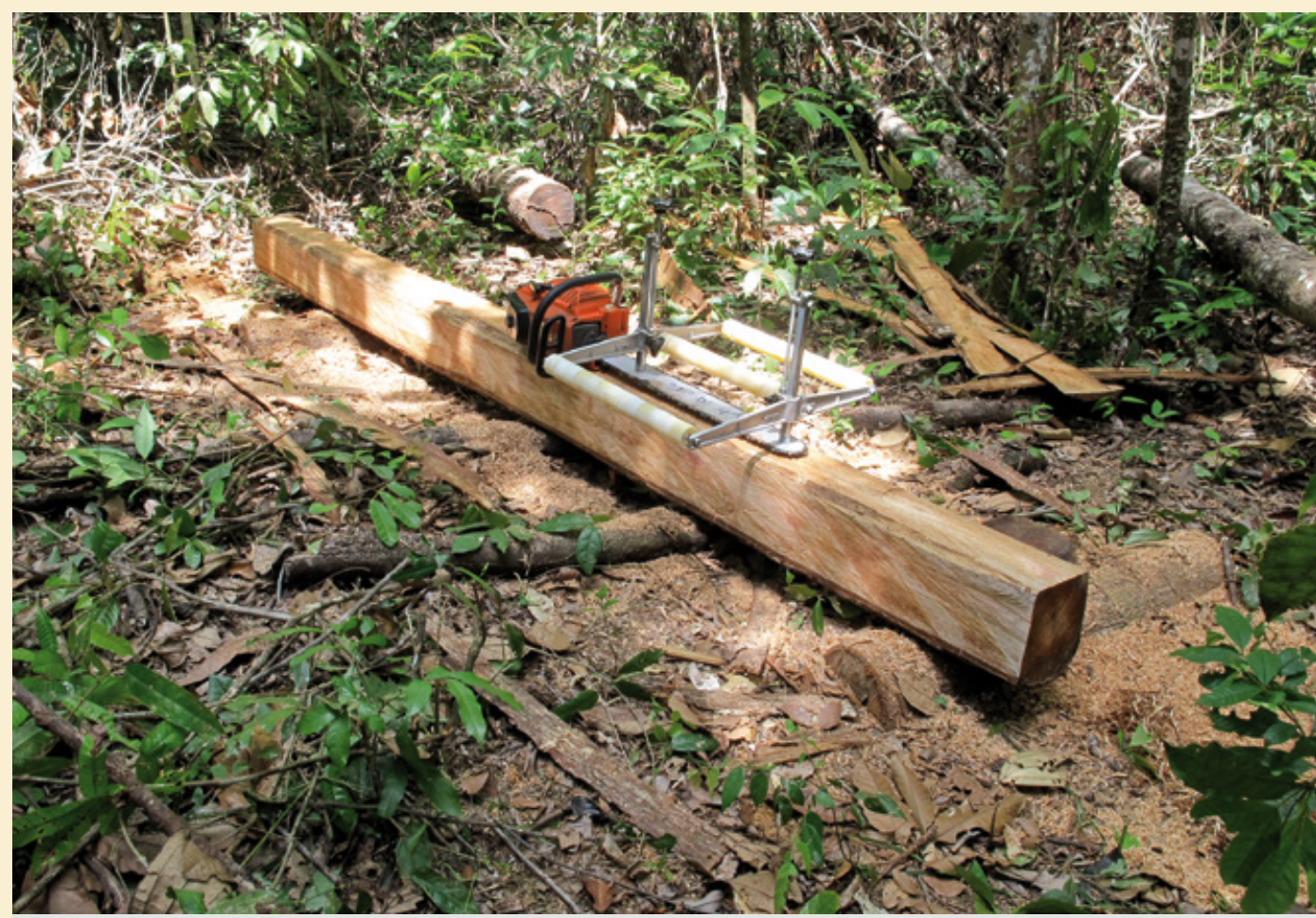

Photo 1.

Sciage d'un Tarrietia utilis de 30 ans dans les plantations de Paracou.

Photo H. Morel. 


\section{RÉSUMÉ}

\section{QUALITÉ ET USAGES DU BOIS DE CINQ ESPĖCES FORESTIÈRES ADAPTÉES À LA PLANTATION À VOCATION DE BOIS D'CEUVRE ET TESTÉES EN GUYANE FRANÇAISE}

Des essais de plantations forestières ont été menés en Guyane française depuis le début des années 1960 sur plus de 138 espèces (70 espèces natives et 68 espèces exotiques). Une étude récente sur la productivité de ces espèces en plantation (projet ForesTreeCulture, 2013-2015) a mis en avant le fort potentiel de production de trois espèces natives (Simarouba amara Aubl., Vochysia tomentosa (G. Mey.) DC., Bagassa guianensis (Aubl.)) et d'une espèce d'Afrique de l'Ouest (Tarrietia utilis Sprague) avec des volumes de bois produits supérieurs à $20 \mathrm{~m}^{3} / \mathrm{ha} / \mathrm{an}$. Cependant, les propriétés du bois de ces espèces commerciales ne sont connues qu'au travers d'arbres issus de forêt naturelle. Nous présentons les propriétés du bois de ces espèces en conditions de plantation - densité, retrait, élasticité, angle du fil, durabilité - et discutons de leurs potentiels et de leurs usages respectifs futurs. Une autre espèce, Cordia alliodora ((Ruiz et Pavon) Oken), a également été retenue bien qu'elle n'ait pas encore été plantée en Guyane française. Cette espèce, native de Guyane, est bien connue en Amérique latine pour son bois et son fort potentiel de croissance en milieu anthropisé.

Mots-clés : propriétés technologiques, qualité du bois, plantation, Guyane française.

\section{ABSTRACT}

\section{QUALITY AND USES OF TIMBER FROM FIVE FOREST SPECIES SUITED TO PLANTATION MANAGEMENT AND TESTED IN FRENCH GUIANA}

Forest plantation trials have been conducted in French Guiana since the early 1960s with over 138 tree species (70 native and 68 exotic). A recent study on their productivity in plantations (ForesTreeCulture project, 2013-2015) showed the high potential of three native species (Simarouba amara Aubl., Vochysia tomentosa (G. Mey.) DC., Bagassa guianensis (Aubl.)) and one West African species (Tarrietia utilis Sprague), all of which produced timber volumes in excess of $20 \mathrm{~m}^{3} /$ ha/year. However, the properties of these as commercial species are known only from trees that have grown in their natural forest environment. This article describes the properties of their timber when produced in plantation conditions - density, shrinkage, elasticity, angle of the grain, durability - and discusses the future potential and uses of each. A fourth species, Cordia alliodora ((Ruiz \& Pavon) Oken), was also selected for study although it has not yet been planted in French Guiana. This species is native to French Guiana and well known across Latin America for its timber and high potential for growth in managed environments.

Keywords: technical properties, timber quality, plantation, French Guiana.

\section{RESUMEN}

\section{CALIDAD Y USOS DE MADERAS DE CINCO ESPECIES FORESTALES PROBADAS EN LA GUAYANA FRANCESA PARA PLANTACIONES DE MADERA DE CONSTRUCCIÓN}

Desde principios de los años 60 , se vienen realizando ensayos de plantaciones forestales en la Guayana Francesa con más de 138 especies (70 especies nativas y 68 exóticas). Un reciente estudio sobre la productividad en plantaciones de dichas especies (proyecto ForesTreeCulture, 2013-2015) destacó el gran potencial productivo de tres especies nativas (Simarouba amara Aubl., Vochysia tomentosa (G. Mey.) DC., Bagassa guianensis (Aubl.)) y una especie de África Occidental (Tarrietia utilis Sprague), con volúmenes de producción de madera superiores a $20 \mathrm{~m}^{3} / \mathrm{ha} / \mathrm{año}$. No obstante, las propiedades de la madera de estas especies comerciales sólo se conocen a través de árboles procedentes de bosques naturales. Presentamos aquí las propiedades madereras de estas especies en condiciones de plantación -densidad, contracción, elasticidad, ángulo de la fibra y durabilidad-y analizamos su potencial y sus respectivos usos futuros. Se seleccionó una especie más, Cordia alliodora ((Ruiz y Pavón) Oken), aunque nunca se ha sembrado en la Guayana Francesa. Esta especie, originaria de Guyana, es bien conocida en América Latina por su madera y su alto potencial de crecimiento en medios antropizados.

Palabras clave: propiedades tecnológicas, calidad de la madera, plantación, Guayana Francesa. 


\section{Introduction}

La population en Guyane est en constante augmentation en raison d'un fort taux de croissance naturelle couplé à une immigration importante. Estimée à 254541 habitants en 2015 , la population guyanaise aura augmenté de $33 \%$ en 2020 et doublée en 2030, d'après les scénarios prospectifs de l'INSEE. La Guyane française devra faire face à une forte augmentation de la demande en bois, que ce soit à vocation de matériau de construction ou énergétique. Le bois utilisé actuellement provient de l'exploitation de la forêt naturelle ou, dans une moindre mesure, est importé depuis la métropole. Ces deux sources d'approvisionnement présentent des contraintes, comme le coût croissant de l'exploitation de la forêt domaniale, ou les coûts de transport et le bilan carbone associé, liés aux importations. La mise en place d'une filière locale de production de bois issus de plantations forestières serait une solution complémentaire à la gestion productive de la forêt naturelle, notamment par la capacité de fourniture régulière d'une quantité significative de bois moins divers, pour les usages standards. En diminuant les impacts sur les forêts naturelles, les plantations forestières sont une des solutions garantissant une gestion durable des écosystèmes forestiers tropicaux (Marien et Mallet, 2004 ; Guitet, 2005 ; Pirard et al., 2016). Les plantations forestières, à vocation de bois d'œuvre ou de bois énergie, sont la principale source d'approvisionnement en bois pour des pays comme le Brésil ou la Chine.

En Guyane, des essais expérimentaux de plantation sur plus de 138 espèces (Hueber et Mauranges, 1984ab ; Bouillet et al., 1985 ; Bouillet et Hueber, 1985 ; Sutter et Bouillet, 1985 ; De Chatelperron et Hueber, 1987 ; Hueber, 1992) ont été lancés dès les années 1960, par le BAFOG (Bureau agricole et forestier guyanais), puis par le CTFT (Centre technique forestier tropical) et l'ONF (Office national des forêts). Les surfaces couvertes par ces essais représentent plus de 800 ha (Nicolini et Morel, 2016). Les espèces testées étaient, en majorité, des espèces locales destinées au bois d'œuvre ou à la trituration. Ces expérimentations, uniques à notre connaissance pour la région amazonienne, représentent une source d'information quant au potentiel des espèces locales mobilisables en plantation.

Dans le cadre d'un projet FEDER ForesTreeCulture 20132015, une première étude a été menée sur ces dispositifs afin d'évaluer la productivité de certaines de ces espèces en plantation. Cette étude a notamment mis en avant le fort potentiel de production de trois espèces natives (Simarouba amara Aubl., Vochysia tomentosa (G. Mey.) DC., Bagassa guianensis (Aubl.)) et d'une espèce exotique (Tarrietia utilis Sprague) d'Afrique de l'Ouest, avec des volumes de bois produits supérieurs à $20 \mathrm{~m}^{3}$ / ha/an (Nicolini et Morel, 2016).

Cependant, les propriétés du bois de ces espèces commerciales ne sont connues qu'au travers d'arbres issus de forêt naturelle (Normand, 1966 ; Dupuy et Chézeaux, 1994). Une seconde étude, dont les résultats sont présentés dans cet article, a donc eu pour but, dans un premier temps, d'étudier les propriétés du bois de ces espèces commerciales en conditions de plantation - densité, retrait, élasticité, angle du fil et durabilité. Une autre espèce, Cordia alliodora ((Ruiz et Pavon) Oken), a également été retenue bien qu'elle n'ait pas encore été plantée en Guyane française. Cette espèce, native de Guyane, est bien connue en Amérique latine pour son bois et son fort potentiel de croissance en milieu anthropisé. Dans un second temps, à partir de ces propriétés et du contexte socio-économique guyanais, les usages de ces bois les mieux appropriés et les plus susceptibles de créer des filières locales rentables et dimensionnées au contexte et au marché guyanais ont été discutés. Par ailleurs, par leur robustesse en plantation, leur bonne vitesse de croissance, et surtout la qualité technologique de leur bois, ces espèces pourraient également constituer des modèles intéressants pour renforcer l'offre mondiale du bois d'œuvre provenant d'espèces de plantations tropicales humides.

\section{Matériel et méthodes}

\section{Site d'étude et échantillonnage}

À l'exception de C. alliodora, échantillonnée sur la

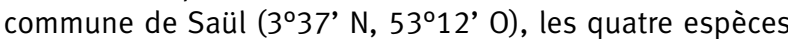
forestières étudiées ont été prélevées dans les plantations de la station expérimentale de Paracou (5¹7' N, 5254' O), mise en place en 1984.

Afin de nous affranchir des singularités induites par des défauts dus à la conformation ou à l'état sanitaire des individus, seuls les arbres ayant un tronc droit, une fourche haute et ne présentant aucun signe de maladie ou d'attaque d'insectes ont été retenus. Cinq individus par espèce ont été abattus, d'un diamètre allant de 30 à $48 \mathrm{~cm}$, et d'une hauteur comprise entre 20 à $30 \mathrm{~m}$ (tableau I).

Pour chaque arbre, une rondelle (figure 1a) a été prélevée à trois niveaux de hauteur sur le tronc : à 1,30 m (hors empâtement, R1), à mi-hauteur entre le sol et la fourche maîtresse (R2) et juste sous l'insertion de la fourche maîtresse (R3). Quelques heures après l'abattage, un barreau radial ( $R \times T \times L=$ diamètre $\times 30 \times 30 \mathrm{~mm}^{3}$; figure $1 \mathrm{~b}$ ) a été découpé à partir de chaque rondelle. Deux billons de $80 \mathrm{~cm}$ de longueur (figure 1d, B1 et B2) ont également été prélevés de part et d'autre de la rondelle R1. Enfin, un plateau diamétral ( $R \times T \times L=D B H \times 50 \times 500 \mathrm{~mm}^{3}$; figure 1e) a été découpé dans chacun des billons.

\section{Mesures physiques et mécaniques}

\section{Infradensité et humidité verte}

À partir de chaque barreau radial (figure $1 \mathrm{~b}$ ), des éprouvettes ont été découpées longitudinalement $(R \times T \times L$ $=10 \times 30 \times 30 \mathrm{~mm}^{3}$; figure 1c). À l'aide d'une balance Sartorius CP224S (précision 0,2 mg), chaque éprouvette a fait l'objet d'une mesure (1) de sa masse verte après découpe par fendage (Mv), (2) de la masse de l'eau déplacée (Mi) par l'éprouvette immergée, et (3) de sa masse anhydre (MO\%) après 48 heures de séchage à $103^{\circ} \mathrm{C}$. L'infradensité (ID) et l'humidité verte ( $\mathrm{Hv}$, en \%) ont été calculées à partir des formules suivantes:

$\mathrm{ID}=\mathrm{M} 0 \% /(\mathrm{Mv}-\mathrm{Mi})=\mathrm{M} 0 \% /$ Vsaturé

$\mathrm{Hv}=(\mathrm{Mv}-\mathrm{MO} \%) / \mathrm{MO} \%$ 


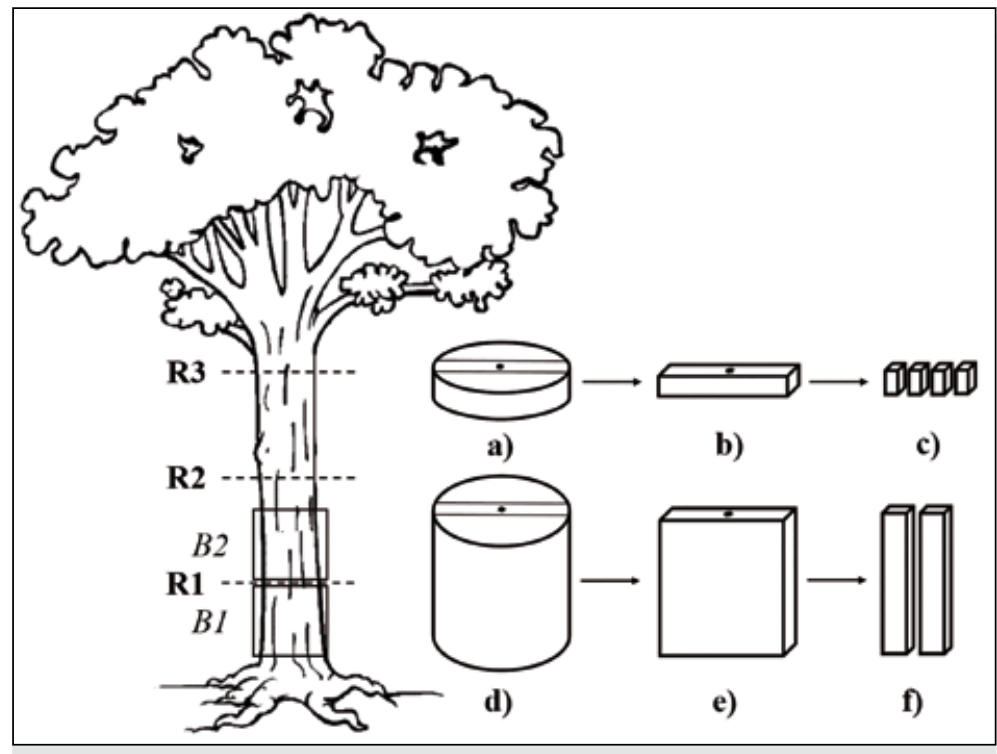

Figure 1.

Schéma d'échantillonnage.
Retrait dimensionnel et point de saturation des fibres (PSF)

À partir d'une partie $(L=100 \mathrm{~mm})$ du plateau issu du billon B2 (figure 1e), deux types d'éprouvette ont été découpés longitudinalement : des échantillons longs $(R \times T \times L=$ $20 \times 20 \times 80 \mathrm{~mm}^{3}$; figure 1f) servant à la mesure du retrait longitudinal et des échantillons courts $(R \times T \times L=20 \times 20$ $x 10 \mathrm{~mm}^{3}$ ) permettant les mesures des retraits radiaux et tangentiels. Chaque éprouvette a été stabilisée successivement, pendant plusieurs semaines, dans quatre ambiances hygrométriques différentes : saturée (18\%, $12 \%, 5 \%)$ et anhydre ( $0 \%$ ) (tableau II). Pour chaque palier de stabilisation, chaque éprouvette a été mesurée $(E)$ dans ses trois dimensions radiale $(r)$, tangentielle (t) et longitudinale (l), grâce à des comparateurs (Mitutoyo ID-H0530 et 543401b) et pesée. Quatre types de retraits totaux (R, en \%) ont été calculés selon les formules suivantes:

$\mathrm{Rr} / \mathrm{Rt} / \mathrm{Rl}=(\mathrm{Er} / \mathrm{Et} / \mathrm{El}$ saturée $-\mathrm{Er} / \mathrm{Et} / \mathrm{El} 0 \%) / \mathrm{Er} / \mathrm{Et} / \mathrm{El}$ saturée $\mathrm{X} 100$

$\mathrm{Rv}=((\mathrm{Er} \times \mathrm{Et} \times \mathrm{El}$ saturée $)-(\mathrm{Er} \times \mathrm{Et} \times \mathrm{El0} \%)) /(\mathrm{Er} \times \mathrm{Et} \times \mathrm{El}$ saturée) $\times 100$

Le point de saturation des fibres (PSF) correspond à l'humidité théorique à laquelle le bois, soumis au séchage, commence à se rétracter. Il est égal à l'abscisse à l'origine de la droite de régression du retrait volumique en fonction de l'état hygroscopique de l'échantillon (tableau II).

Tableau I.

Diamètre, hauteur totale et largeur d'aubier des cinq individus étudiés de Simarouba amara, Vochysia tomentosa, Cordia alliodora, Tarrietia utilis et Bagassa guianensis.

\begin{tabular}{|l|l|l|l|}
\hline Espèces & Diamètre $\mathbf{( c m )}$ & Hauteur $(\mathbf{m})$ & Aubier $(\mathbf{c m})$ \\
\hline Simarouba amara & & & \\
\hline Vochysia tomentosa & $41,49,47,40,43$ & $27,29,29,28,29$ & $3,4,3,4,4$ \\
\hline Cordia alliodora & $41,43,40,40,32$ & $24,25,25,24,23$ & $4,6,2,5,4$ \\
\hline Tarrietia utilis & $25,25,52,57,25$ & $25,29,30,30,27$ & $1,3,5,1,1$ \\
\hline Bagassa guianensis & $49,34,32,30,27$ & $30,24,24,24,20$ & $2,2,3,2,2$ \\
\hline
\end{tabular}

\begin{tabular}{|c|c|c|c|c|c|}
\hline \multicolumn{6}{|c|}{$\begin{array}{l}\text { Tableau II. } \\
\text { Humidités moyennes (en \%) de stabilisation des bois pour les différentes espèces étudiées en fonction } \\
\text { des différentes ambiances d'exposition. L'écart-type est entre parenthèses. }\end{array}$} \\
\hline \multirow[b]{2}{*}{ Simarouba amara } & $\begin{array}{c}\text { Étuve à } 103^{\circ} \mathrm{C} \\
\text { pendant } 48 \mathrm{~h}\end{array}$ & $\begin{array}{l}\text { Étuve à } 45^{\circ} \mathrm{C} \\
\text { pendant } \\
1 \text { mois }\end{array}$ & $\begin{array}{l}\text { Pièce climatisée } \\
\quad>6 \text { semaines } \\
\left(\mathrm{T}=25^{\circ} \mathrm{C} ; \mathrm{H}=62 \%\right)\end{array}$ & $\begin{array}{l}\text { Pièce non climatisée } \\
\qquad \begin{array}{l}\left(T=28^{\circ} \mathrm{C} ;\right. \\
H=80 \%)\end{array}\end{array}$ & $\begin{array}{l}\text { Échantillons } \\
\text { verts conservés } \\
\text { dans l'eau }\end{array}$ \\
\hline & 0,0 & $5,0(0,5)$ & $12,2(1,3)$ & $14,8(1,6)$ & $136,4(30,4)$ \\
\hline Vochysia tomentosa & 0,0 & $5,8(0,5)$ & $12,9(1,4)$ & $16,6(1,8)$ & $192,5(33,6)$ \\
\hline Cordia alliodora & 0,0 & $5,3(1,4)$ & $10,6(1,4)$ & - & $90,8(50,4)$ \\
\hline Tarrietia utilis & 0,0 & $5,2(0,3)$ & $12,7(0,6)$ & $16,6(1,0)$ & $68,5(15,9)$ \\
\hline Bagassa guianensis & 0,0 & $4,9(0,5)$ & $11,1(0,8)$ & $13,8(1,3)$ & $82,7(30,7)$ \\
\hline
\end{tabular}




\section{Module d'élasticité (MOE) et module spécifique (MOESP)}

À partir de l'autre partie du plateau issu du billon B2 (figure 1d), des éprouvettes ( $R \times T \times L=V=18 \times 18 \times$ $360 \mathrm{~mm}^{3}$; figure 1f) ont été découpées longitudinalement $(L=400 \mathrm{~mm})$. Chaque éprouvette préalablement stabilisée à $12 \%$ d'humidité dans une salle climatisée a été mesurée et pesée (M12\%) afin de calculer sa densité (D12 = M12\% / V). Le module d'élasticité (MOE, en GPa) a été estimé par la méthode BING $\circledast$ (Beam Identification by Non destructive Grading ; Baillères et al., 1998). Le module spécifique $(M O E S P=$ MOE / D12, en GPa) a également été calculé.

\section{Angle du fil}

L'angle du fil a été mesuré visuellement à l'aide d'un goniomètre, à partir des échantillons ayant servi aux mesures d'infradensité et d'humidité verte. Pour cela, les faces LR et RT de l'échantillon ont été plaquées sur le bâti de l'appareil de mesure. La déviation des fibres a été mesurée sur la face LT par rapport à la face de débit LR qui correspondait approximativement à l'axe principal de la tige.

\section{Essais de durabilité}

Le protocole d'essai permettant de mesurer la durabilité naturelle des bois est basé sur la norme européenne XP CEN/TS 15083-2. Ce protocole consiste à estimer la durée de vie de fines éprouvettes en contact avec un humus forestier maintenu humide. Pour chaque arbre, 18 éprouvettes $(R \times T \times L$ $=10 \times 5 \times 100 \mathrm{~mm}^{3}$ ), représentant trois répétitions par mois durant les six mois d'expérimentation, ont été prélevées dans chacune des trois zones déterminées : l'aubier en périphérie et proche de l'écorce, le duramen externe situé près de la transition aubier/duramen, et le duramen interne proche de la moelle. Ces éprouvettes ont été découpées dans le billon B1 (figure 1e). Chaque éprouvette, d'abord mise à sécher pendant 48 heures à $103^{\circ} \mathrm{C}$, a été pesée puis placée dans un bac de terre. Ces 18 éprouvettes étaient associées à six éprouvettes témoins de référence de Virola michelii uniformément réparties dans les bacs. Chaque mois, neuf éprouvettes par individu (trois zones $x$ trois répétitions), ainsi qu'une éprouvette témoin, ont été prélevées, séchées à $103^{\circ} \mathrm{C}$ pendant 48 heures, puis pesées. Une perte de masse a ainsi été obtenue pour chaque éprouvette. Pour chaque espèce, la perte de masse relative d'une espèce a été calculée à partir du rapport de la médiane de la perte de masse du dernier prélèvement et de la perte de masse médiane de l'essence témoin de référence.

Deux classements de dégradation sont proposés. Le premier classement (intra-bac) compare la dégradation de l'échantillon à celle de l'essence témoin du même bac. Le second classement (inter-bac) compare la dégradation de l'échantillon à celle de l'essence témoin de référence moyenne tous bacs confondus.

\section{Analyses des données}

Une description statistique générale (médiane, premier et troisième quartile, minimum et maximum) a été réalisée pour l'ensemble des propriétés du bois des cinq espèces. Le test de Kruskal-Wallis a été utilisé pour comparer les différents groupes. Lorsque ce test était significatif, il était suivi d'un test post hoc de comparaisons multiples. Pour le descripteur de la durabilité naturelle, des régressions linéaires ont été effectuées afin d'obtenir une perte de masse à six mois, calculée à partir de toutes les valeurs mesurées durant les six mois de prélèvement. Toutes les analyses ont été effectuées avec le logiciel R (R Development Core Team, 2011).

\section{Résultats}

\section{Propriétés physiques et mécaniques moyennes du bois des espèces étudiées}

\section{Densité}

Toutes espèces confondues, les densités moyennes varient du simple au double (figure 2). Trois espèces ont un bois assez léger (S. amara, V. tomentosa et $C$. alliodora), avec une densité moyenne à $12 \%$ comprise entre 0,4 et 0,5 . T. utilis a une densité moyenne à $12 \%$ intermédiaire de 0,65 , tandis que $B$. guianensis a en moyenne le bois le plus dense à $12 \%$ avec une valeur de 0,8 . Contrairement à $C$. alliodora et $B$. guianensis, les trois essences $S$. amara, $V$. tomentosa et $T$. utilis présentent des densités intra-espèce et intra-individu assez homogènes. Une inversion de classement entre densité à $12 \%$ et infradensité est observée pour S. amara et V. tomentosa. Cette inversion est le fait de la forte rétraction de $V$. tomentosa au séchage.

\section{Humidité verte}

L'humidité verte moyenne varie fortement en fonction des espèces. $V$. tomentosa et $C$. alliodora possèdent une humidité verte moyenne très élevée ( $\mathrm{Hv}>100 \%$ ), indiquant que ces deux espèces peuvent contenir jusqu'à trois fois plus d'eau que de bois en volume et sont caractérisées par une forte variabilité intra-espèce et intra-individu. À l'inverse, $S$. amara et $T$. utilis présentent une humidité verte moyenne plus faible d'environ $65 \%$ et moins variable.

\section{Angle du fil}

Seul $B$. guianensis possède un angle du fil prononcé et variable pouvant atteindre $30^{\circ}$ par rapport à l'axe du tronc (figure 3). Cet angle s'exprime sous forme de contrefil. Le contrefil est dû à une inclinaison variable mais régulière de l'angle du fil d'une couche de bois à l'autre à mesure que celles-ci sont formées au cours de la croissance de l'arbre. Ce contrefil a une période d'environ $4 \mathrm{~cm}$, c'est-à-dire que, tous les $4 \mathrm{~cm}$ environ, le fil du bois retrouve une même valeur. L'amplitude des variations de l'angle du fil est assez faible lorsque l'arbre est juvénile, et s'amplifie largement avec le rayon lorsque l'arbre est plus mature. $V$. tomentosa peut avoir un angle du fil qui dépasse localement $10^{\circ}$, sous la forme d'un léger contrefil à large période d'environ $5 \mathrm{~cm}$, tandis que S. amara, T. utilis et C. alliodora ont un angle du fil faible $\left({ }^{\circ} 1^{\circ}\right)$.

\section{Retraits}

La variabilité du retrait volumique (Rv) est principalement influencée par le retrait tangentiel (Rt). S. amara et $B$. guianensis présentent de faibles valeurs de retrait $(\mathrm{Rv}=7 \% ; \mathrm{Rt}=4,5 \%), T$. utilis et $C$ alliodora affichent des 

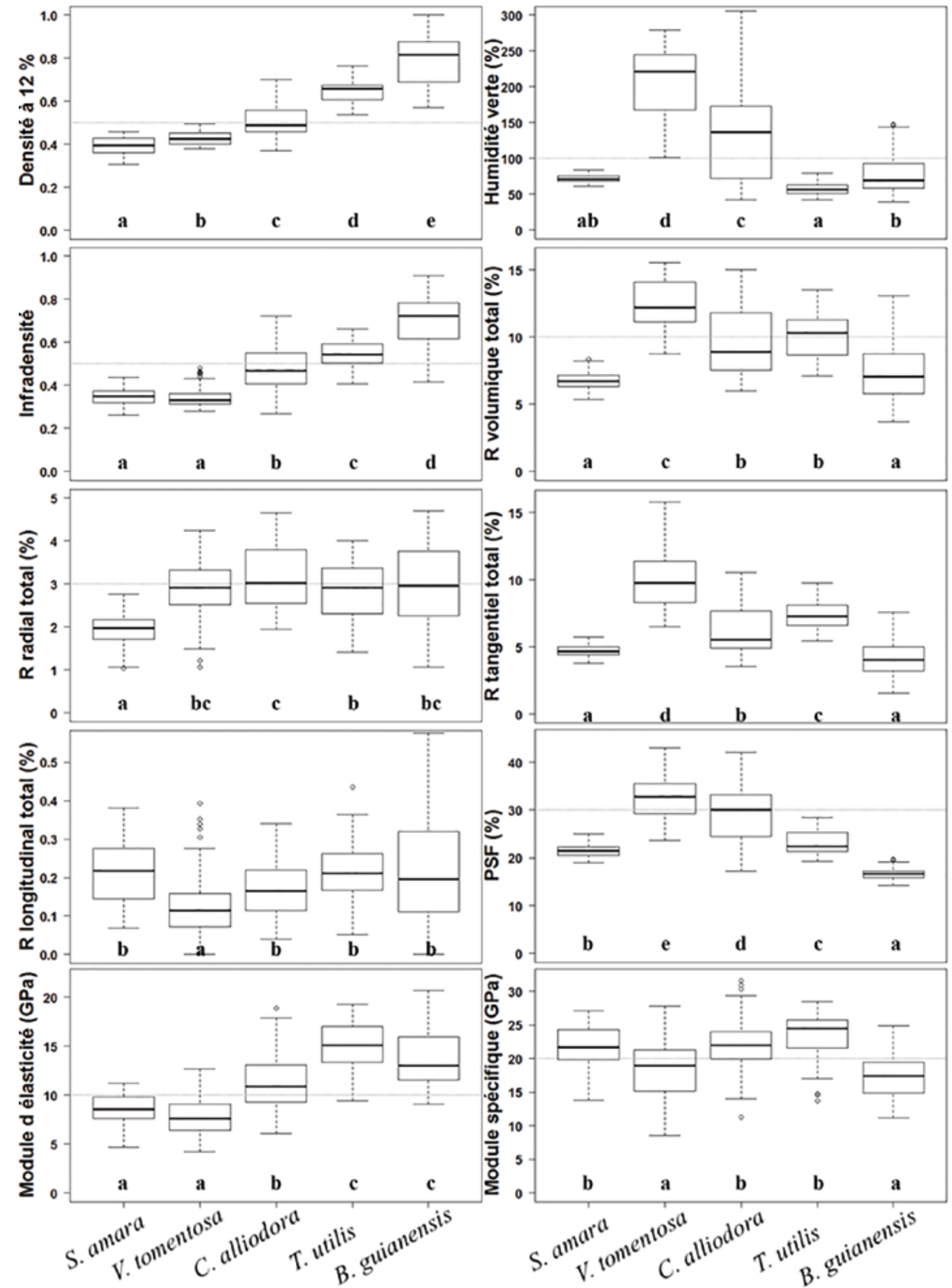

Figure 2.

Variations des propriétés physiques et mécaniques du bois chez les cinq espèces étudiées. Un test de Kruskal-Wallis est utilisé pour différencier les espèces. Lorsqu'il est significatif, il est suivi par un test post hoc de comparaisons multiples. Deux espèces avec la même lettre ne sont pas significativement différentes ( $P>0,05)$. 


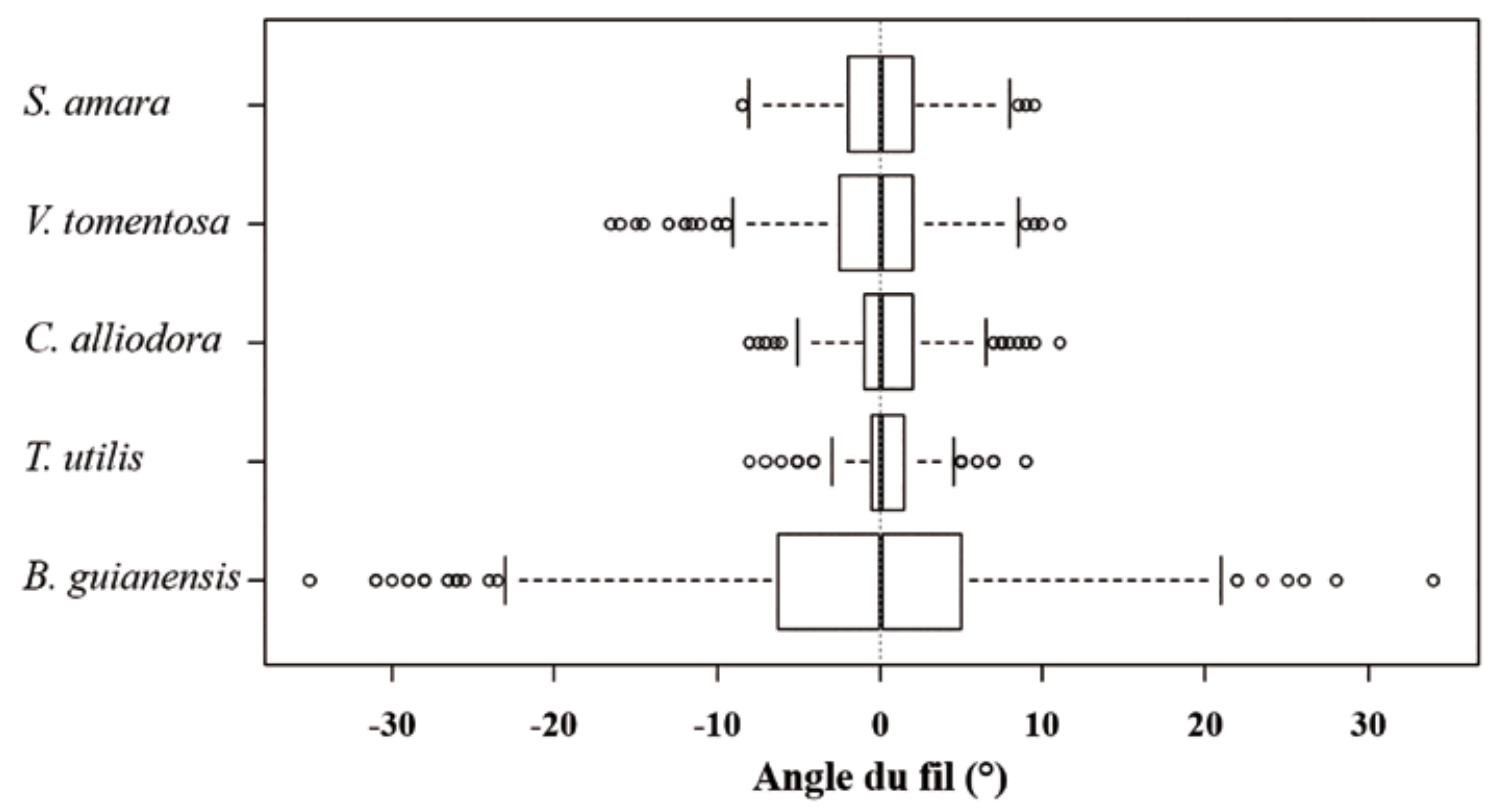

Figure 3.

Variation de l'angle du fil pour les cinq espèces étudiées.

valeurs intermédiaires ( $R v=10 \%$; Rt $=6,5 \%$ ), tandis que les plus fortes valeurs sont observées chez $V$. tomentosa $(\mathrm{Rv}=12 \% ; \mathrm{Rt}=6,5 \%)$. Les retraits radiaux ( $\mathrm{Rr})$ et longitudinaux (Rl) sont relativement homogènes entre les cinq espèces, avec respectivement $3 \%$ et $0,2 \%$ en moyenne, excepté pour $S$. amara qui présente un retrait radial moyen plus faible autour de $2 \%$.

\section{Point de saturation des fibres (PSF)}

La gamme de variabilité du PSF est grande chez les espèces étudiées puisqu'elle va de $16 \%$ chez $B$. guianensis à au moins $30 \%$ chez C. alliodora (30\%) et V. tomentosa (32\%). Les valeurs intermédiaires autour de $20 \%$ sont observées chez S. amara (21\%) et T. utilis (23\%).

\section{Module d'élasticité (MOE) et module spécifique (MOESP).}

La gamme du module d'élasticité couverte par les cinq espèces est également importante. S. amara et $V$. tomentosa ont un MOE moyen de $8 \mathrm{GPa}$ alors que $T$. utilis et $B$. guianensis ont un MOE moyen de $15 \mathrm{GPa}$. En revanche, le module spécifique (MOESP) des cinq espèces couvre une gamme plus restreinte. Ce sont les espèces à fort angle du fil, $V$. tomentosa et $B$. guianensis, qui ont les valeurs moyennes de MOESP les plus faibles, autour de $18 \mathrm{GPa}$, tandis que les trois autres espèces présentent des valeurs moyennes plus élevées, de 23 à 24 GPa.

\section{Durabilité}

La durabilité naturelle estimée dans l'essai « sol » donne un classement légèrement différent en fonction du type de référence témoin intra-bac ou inter-bacs (figure 4). En ce qui concerne le duramen, S. amara possède la durabilité la plus faible (classes 3 ou 4 selon le référencement). Les quatre autres espèces ont une durabilité moyennement durable à durable (classes 2 ou 3 selon le référencement). On remarque en outre que trois essences (S. amara, $T$. utilis et $B$. guianensis) ont un aubier plus résistant (classes 3 ou 4 selon le référencement) que les deux autres.

\section{Variation spatiale des propriétés physiques et mécaniques du bois des espèces étudiées}

\section{Variation de l'infradensité le long du tronc}

Au sein d'un individu, S. amara, V. tomentosa et, dans une moindre mesure, $C$. alliodora présentent une augmentation de leur infradensité moyenne de la base du tronc vers la partie sommitale sous la couronne (tableau III, annexe 1). Chez T. utilis, l'évolution est plus complexe, avec une diminution de l'infradensité moyenne depuis la base jusqu'à la moitié du tronc, suivie d'une augmentation depuis la mi-hauteur jusqu'à l'insertion de la couronne. Enfin, B. guianensis ne présente pas de gradient d'infradensité moyenne le long du tronc.

\section{Variation radiale des propriétés}

Les profils radiaux présentent une augmentation de l'infradensité de la moelle vers l'écorce chez $C$. alliodora et $T$. utilis (annexe 2). Chez B. guianensis, après une augmentation, l'infradensité diminue lorsqu'on se rapproche de l'écorce (figure 5, annexe 1). À l'inverse, chez S. amara et $V$. tomentosa, on observe une légère diminution, suivie d'une augmentation (figure 5, annexe 1).

En ce qui concerne le retrait, le MOE et le MOESP, il n'y a pas de variations radiales notables, sauf dans des cas particuliers liés au développement de l'arbre et à la formation de bois de tension. 

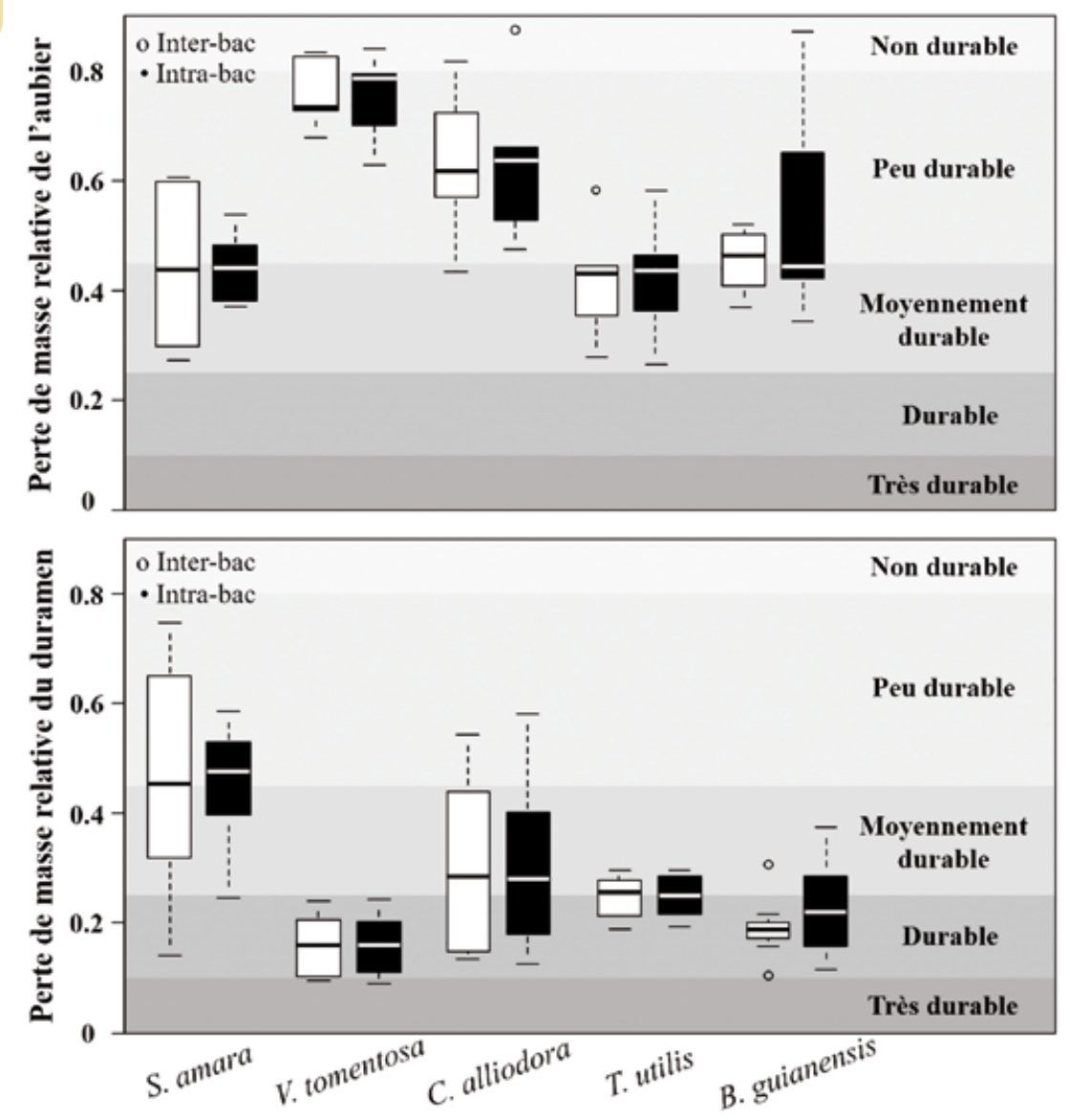

Figure 4.

Perte de masse relative et classes de durabilité basées sur la norme européenne XP CEN/TS 15083-2 de l'aubier et du duramen pour les cinq espèces étudiées. Le duramen comprend le duramen externe situé près de la transition aubier/ duramen, et le duramen interne proche de la moelle puisqu'il n'y a pas de différence significative entre ces deux parties en ce qui concerne ce caractère.

\section{Bois de tension}

Certaines conditions de croissance obligent l'arbre à mettre en place un bois particulier dit bois de tension, qui permet à l'arbre de se redresser et se réorienter lors de sa croissance. Ce type de bois possède des propriétés mécaniques et physiques différentes du bois normal (Clair et Thibaut, 2014). La figure 6 en présente un bon exemple avec un individu de S. amara non inclus dans les observations précédentes. L'excentrement marqué de cet individu, ainsi qu'une importante augmentation du retrait volumique et du module d'élasticité, nous permettent de confirmer la présence de bois de tension le long du plus grand rayon. Du bois de tension a également été observé chez $V$. tomentosa et $B$. guianensis.

\section{Différences entre aubier et duramen}

Pour l'ensemble des espèces étudiées, il n'y a pas de variabilité des propriétés physiques et mécaniques marquée entre aubier et duramen, excepté pour les retraits et les PSF de $C$. alliodora et $B$. guianensis (tableau IV). La durabilité de l'aubier, quant à elle, est toujours plus faible pour les quatre espèces présentant un duramen différencié (figure 4).

\section{Tableau III.}

Infradensité moyenne en fonction des hauteurs pour les cinq espèces étudiées. L'écart-type est entre parenthèses. Par espèce, un test de Kruskal-Wallis est utilisé pour différencier les hauteurs. Lorsqu'il est significatif, il est suivi par un test post hoc de comparaisons multiples. Deux hauteurs au sein d'une même espèce avec la même lettre ne sont pas significativement différentes $(P>0,05)$.

\begin{tabular}{|c|c|c|c|c|}
\hline Nom local & Espèces & DBH & Mi-tronc & Sous la fourche \\
\hline Simarouba & Simarouba amara & $\begin{array}{c}0,35(0,035) \\
a\end{array}$ & $\begin{array}{c}0,37(0,036) \\
b\end{array}$ & $\begin{array}{c}0,38(0,029) \\
c\end{array}$ \\
\hline Wana Kouali & Vochysia tomentosa & $\begin{array}{c}0,34(0,038) \\
a\end{array}$ & $\begin{array}{c}0,36(0,039) \\
b\end{array}$ & $\begin{array}{c}0,39(0,030) \\
c\end{array}$ \\
\hline Cèdre Sam & Cordia alliodora & $\begin{array}{c}0,47(0,098) \\
a\end{array}$ & $\begin{array}{c}0,52(0,11) \\
b\end{array}$ & $\begin{array}{c}0,56(0,10) \\
c\end{array}$ \\
\hline Niangon & Tarrietia utilis & $\begin{array}{c}0,55(0,060) \\
a\end{array}$ & $\begin{array}{c}0,51(0,052) \\
b\end{array}$ & $\begin{array}{c}0,53(0,036) \\
c\end{array}$ \\
\hline Bagasse & Bagassa guianensis & $\begin{array}{c}0,70(0,11) \\
a\end{array}$ & $\begin{array}{c}0,68(0,11) \\
b c\end{array}$ & $\begin{array}{c}0,70(0,085) \\
a b\end{array}$ \\
\hline
\end{tabular}




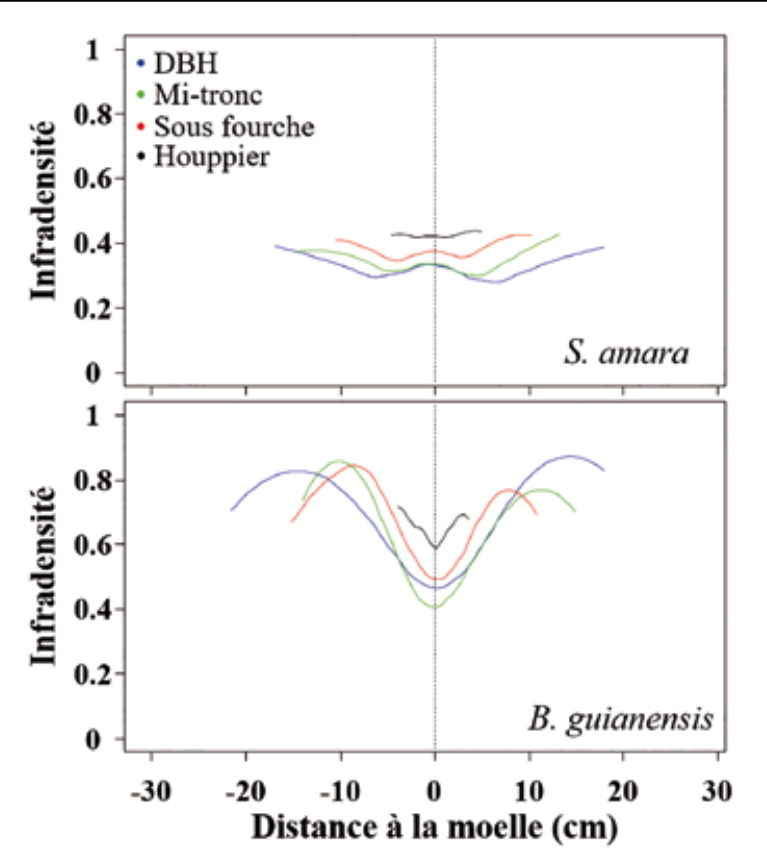

Figure 5.

Profils moyens radiaux (en fonction de la distance à la moelle) et longitudinaux (en fonction de la hauteur dans l'arbre) d'infradensité chez S. amara (en haut) et $B$. guianensis (en bas). Le trait vertical en pointillé représente la moelle.

\section{Comparaison des résultats aux valeurs de référence provenant d'arbres de forêt naturelle}

En général, les résultats montrant des valeurs un peu plus faibles que celles mesurées sur le bois d'arbres adultes de forêt naturelle, il est fort probable qu'il y ait avant tout un effet de la juvénilité des bois que nous avons récoltés. Concernant les résultats sur la durabilité aux champignons, le bois de $S$. amara montre une résistance un peu plus forte que ce qui a été mesuré dans les essais suivant la norme XP CEN/TS 15083-1 ; les deux méthodes d'essai étant différentes, il est assez normal que certaines essences ne réagissent pas de la même façon. Depuis 20 ans que notre laboratoire mesure la dégradation de $S$. amara sur lit de sol forestier, cette différence est systématique sur cette espèce. Une synthèse des résultats comparatifs est présentée en annexe 2.

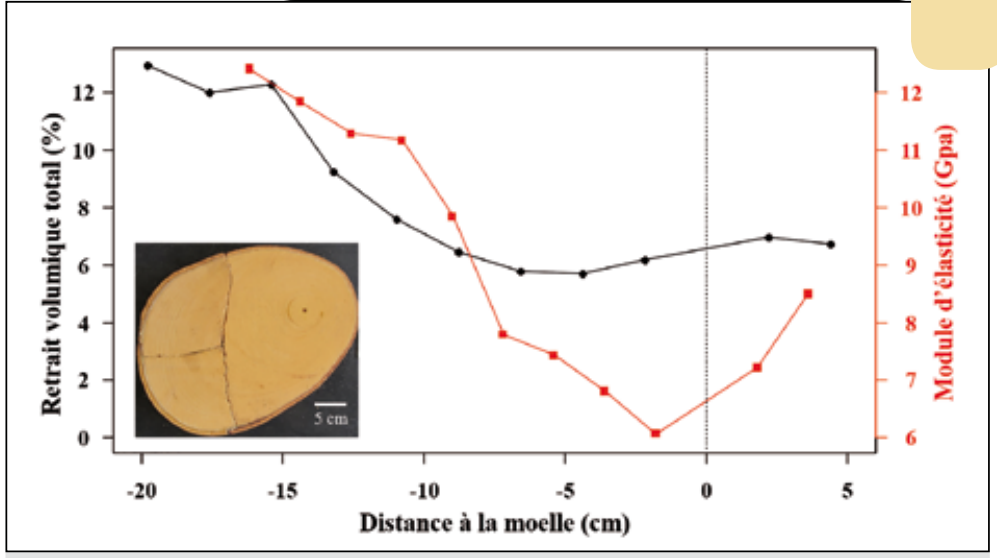

Figure 6.

Profils moyens radiaux du retrait volumique total (\%, en noir) et du module d'élasticité (GPa, en rouge) chez un $S$. amara très excentré. Le trait vertical en pointillé représente la moelle. En bas à gauche, la photo de la rondelle correspondante.

\section{Discussion}

\section{Cinq essences à fort potentiel}

Cette étude a permis de mettre en avant les propriétés du bois des cinq espèces sélectionnées pour leur réussite en plantation en Guyane. Elle montre une grande dispersion des propriétés technologiques mesurées, avec des grandeurs pouvant atteindre des facteurs de 2 à 3 entre les différentes essences, mais aussi une forte variabilité dans l'arbre de certaines propriétés comme l'infradensité. Si ce large panel de propriétés présage un fort potentiel de valorisation, il nécessite toutefois une parfaite connaissance du patron des propriétés à l'intérieur de l'arbre et de la variabilité existante entre individus, de manière à optimiser l'usage de ces espèces après exploitation.

\section{Une propriété d'avenir pour les arbres de plantation : la durabilité naturelle}

Parmi les propriétés évaluées dans cette étude, la durabilité naturelle est la plus importante. En effet, les produits de traitement des bois, indispensables à l'utilisation de bois non durables dans les bâtiments en zone tropicale, sont à la fois

\section{Tableau IV.}

Point de saturation des fibres (PSF) et retraits volumique (Rv), tangentiel (Rt), radial (Rr) et longitudinal (Rl) totaux moyens pour l'aubier et le duramen de Cordia alliodora et Bagassa guianensis. L'écart-type est entre parenthèses. Les valeurs en gras signifient que, pour une même espèce, la différence entre l'aubier et le duramen est significativement différente au seuil de $5 \%$ (test de Kruskal-Wallis).

\begin{tabular}{|c|c|c|c|c|c|c|}
\hline Espèces & Type de bois & PSF (\%) & $\operatorname{Rv}(\%)$ & Rt (\%) & $\operatorname{Rr}(\%)$ & RI (\%) \\
\hline \multirow[t]{2}{*}{ Cordia alliodora } & Aubier & $23,7(5,0)$ & $10,6(1,4)$ & $5,7(1,2)$ & $3,0(0,9)$ & - \\
\hline & Duramen & $31,7(5,3)$ & $11,1(0,8)$ & $5,9(1,9)$ & $3,0(0,9)$ & - \\
\hline \multirow[t]{2}{*}{ Bagassa guianensis } & Aubier & $21,9(1,3)$ & $11,3(1,7)$ & $6,4(1,1)$ & $5,0(1,1)$ & $\mathbf{0 , 5}(0,5)$ \\
\hline & Duramen & $16,8(1,5)$ & $6,7(1,6)$ & $3,8(0,9)$ & $\mathbf{2 , 8}(0,9)$ & $\mathbf{0 , 3}(0,3)$ \\
\hline
\end{tabular}


toxiques pour l'environnement, mais aussi de moins en moins efficaces, suite au durcissement des réglementations européennes vis-à-vis de l'usage des métaux lourds. L'opportunité de produire un bois naturellement durable pour le bâtiment représente en ce sens une véritable perspective d'avenir.

Une difficulté, et non la moindre, est d'apprécier et surtout de quantifier correctement la durabilité naturelle d'un bois. La méthode choisie ici se calque sur la norme de l'ENV 807 pour tester la durabilité des bois sélectionnés face aux champignons. Cependant, comme tous les standards qui estiment la durabilité, cette norme a ses limites. L'essai de l'ENV 807 met les éprouvettes de bois en contact avec du sol forestier maintenu à un fort taux d'humidité et contenant une grande diversité de spores de champignons xylophages (Schimann et al., 2017). Dans de telles conditions, ce sont essentiellement des champignons de pourriture molle et de pourriture blanche qui sont mobilisés, tandis que les pourritures brunes ne sont ici pas testées. Cet aspect n'est pas sans importance car les bois de la famille des Vochysiaceae ou bien celui de l'angélique de Guyane (Dicorynia guianensis), même s'ils se révèlent très résistants en essai XP ENV 807 , le sont beaucoup moins lorsqu'ils sont mis en contact avec des champignons de pourriture brune (Longwood, 1962 ; Paradis et al., 2011).

Par ailleurs, les champignons sollicités, présents dans le sol d'essai selon une grande diversité, entrent en concurrence lors de la mise en place des essais. Cette concurrence à la ressource est d'autant plus importante que l'échantillon est appétant. Ainsi, les éprouvettes très appétantes de $V$. michelii (témoin) sont rapidement colonisées par les champignons les plus proches. Du fait de la grande diversité de cinétique de dégradation entre espèces de champignons, la perte de masse finale à six mois des éprouvettes témoins peut varier du simple au double, d'un bac de culture à l'autre, chez certaines espèces comme $S$. amara ou $B$. guianensis. Les méthodes de normalisation de ces tests prennent toute leur importance en ce qui concerne les espèces à durabilité intermédiaire, comme le montre la figure 4. En revanche, pour les espèces plus durables, la perte de masse finale est beaucoup plus homogène, sans doute du fait de la spécialisation enzymatique des champignons.

\section{Quelles essences de plantation pour quels usages en Guyane?}

- Le bois de S. amara sans duramen visible est blanc crème uniforme, tendre et léger. Ses propriétés mécaniques sont plutôt faibles. Il présente malgré tout une assez bonne rigidité, compte tenu de sa faible densité, et surtout un retrait faible. Sa durabilité face aux champignons est plutôt faible. Pourtant, de manière paradoxale, les sondages dans les bâtiments historiques du XIXe et du XXe siècle montrent qu'il était souvent utilisé pour la fabrication de faux-plafonds et planchers de combles (exemple de la cure de l'église d'Iracoubo), en raison de sa bonne résistance aux termites lorsqu'il est mis en œuvre en zone protégée des intempéries (classe d'emploi 2B). Le bois de S. amara est plutôt un bois de déroulage, d'emballage, d'ameublement et de menuiseries intérieures. Toutefois, son usage en charpente légère ou en lamellé-collé peut être envisagé en raison de son bon module spécifique. Par contre, il devra impérativement être protégé des intempéries car le lessivage des métabolites contenus dans son bois entraîne une dégradation rapide. En Guyane, une production industrielle de cette espèce pourrait alimenter une filière de lamellé-collé et/ou la production de menuiserie intérieure, comme les frises et les lambris. L'émergence parallèle d'une filière d'emballage, inexistante actuellement, pour les fruits et légumes locaux est également envisageable. Si sa sylviculture devait émerger, elle devrait faire appel à des bûcherons spécialisés en abattage directionnel puisque la bille de cet arbre en plantation a tendance à s'éclater et se fragmenter lors d'un abattage non contrôlé (Nicolini et Morel, 2016). De telles techniques sont déjà pratiquées et enseignées par les agents du Parc amazonien de Guyane.

- Le duramen de $V$. tomentosa est rose-brun, tendre et léger, serti par un aubier plus clair et relativement large de 2 à $4 \mathrm{~cm}$. Ses propriétés mécaniques sont faibles. Si sa densité est égale à celle de $S$. amara, sa rigidité est plus faible et plus variable. Par ailleurs, sa rétractabilité au séchage est plus importante que celle du S. amara et l'anisotropie du retrait est élevée $(R t / R r=2,7)$. Bien que cette espèce ait donné de bons résultats lors de l'essai de durabilité, celle-ci est décrite comme faible dans les essais spécifiques comme l'EN 350 (Gérard et al., 2016), de même que sa résistance aux termites (Gérard et al., 2016). Toutefois, l'appétence pour le bois sec et sain reste assez faible. Les propriétés de $V$. tomentosa destinent cette espèce aux mêmes usages que ceux de S. amara: déroulage, mobilier et menuiseries intérieures. Son bois peut aussi convenir à la production de lamellé-collé à faible inertie. En raison de son fort retrait, il doit impérativement être séché à un taux d'humidité similaire à celui de l'espace de mise en œuvre et tenu éloigné des intempéries pour éviter de fortes variations dimensionnelles en conditions d'usage. En Guyane, une production industrielle de cette espèce pourrait alimenter une filière de lamellé-collé, la production de mobilier et menuiseries intérieures. Une menuiserie de Kourou a produit des chaises pendant plusieurs années à partir de cette essence. Les sous-produits pourraient aussi convenir à une filière d'emballage.

- Le duramen de C. alliodora est brun clair, assez tendre et plutôt léger bien que sa densité soit variable. Ce duramen est généralement serti par un aubier clair assez large de 7 à $10,5 \mathrm{~cm}$. Ses propriétés mécaniques sont moyennes. Sa rigidité par rapport à sa densité est assez bonne. Sa rétractabilité au séchage est assez faible et l'anisotropie du retrait est moyenne ( $R t / R r=2,1)$. Sa durabilité variable face aux champignons est moyenne à bonne dans notre essai « sol ». Elle est en effet décrite comme assez variable et en relation avec la coloration du bois de cœur, les bois les plus sombres étant les plus résistants (Meier, 2015). Sa résistance aux termites est aussi donnée comme assez bonne (Desalegn, 2012). Le bois de cette espèce a été largement plébiscité en raison de sa large aire de répartition naturelle de l'Argentine au Mexique et de ses propriétés particulièrement attractives. En Guyane, en fonction de la dynamique de croissance ou de la sélection 
génétique, il est possible d'envisager un marché provenant de plantations pour la charpente massive et le lamellé-collé pour les bois de densité à $12 \%$ comprise entre 0,5 et 0,6 . Pour les provenances les plus colorées et plus denses, il est possible d'envisager un marché de bardage et de menuiseries extérieures tandis que les provenances les moins denses pourraient être utilisées en menuiseries d'intérieur.

- T. utilis est une essence d'origine africaine particulièrement robuste en plantation en Guyane, avec un duramen brun, assez dur et de densité moyenne. Ce duramen est serti par un aubier plus ou moins large avec des propriétés variables qui restent à éclaircir. Ses propriétés mécaniques sont bonnes, de même que sa rigidité par rapport à sa densité. Sa rétractabilité au séchage est moyenne, de même que l'anisotropie du retrait $(R t / R r=2,3)$. Sa durabilité face aux champignons est moyenne à bonne dans notre essai " sol », tandis qu'elle est rapportée comme faible face aux termites (Gérard et al., 2016). Le bois de cette espèce n'est toutefois pas attaqué lorsqu'il est mis en œuvre à l'abri des intempéries. La plantation industrielle en Guyane de cette espèce robuste pourrait alimenter un marché de charpente et de lamellé-collé, ainsi qu'un marché du mobilier et de menuiseries intérieures.

- Le duramen de $B$. guianensis est jaune vif frais, devenant brun lorsqu'il est exposé aux rayons ultraviolets. Exception faite du bois juvénile, c'est un bois dur et dense. Ses propriétés mécaniques sont bonnes mais variables sur les pièces de petite section. Sa rigidité par rapport à sa densité est assez faible. Le retrait au séchage comme son anisotropie $(R t / R r=1,5)$ sont faibles. Sa durabilité face aux champignons est bonne dans notre essai « sol ». Il est considéré comme résistant aux termites (Gérard et al., 2016). Avec un aubier plutôt mince d'environ $2,5 \mathrm{~cm}$, c'est un bois particulièrement attractif. Cependant, son débit et son séchage doivent être maîtrisés. La présence d'un contre-fil prononcé fait qu'après débit les sciages sont sensibles aux déformations. De plus, la présence de secteur de bois de tension à couche $G$ partiellement lignifiée (Ghislain et Clair, 2017) peut induire des éclatements lors de séquences de séchage trop intenses. Si le séchage et le débit de sciage sont bien optimisés, une plantation industrielle de cette espèce en Guyane pourrait alimenter une filière de charpente et de lamellé-collé, mais aussi la production de bardage, de menuiserie extérieure ainsi que de mobilier urbain.

\section{L'importance de la valorisation des aubiers en plantation}

Actuellement, l'exploitation à faible impact du domaine forestier permanent de Guyane ne permet pas une valorisation optimale des aubiers des essences exploitées, étant donné leur faible durabilité et les longues périodes de stockage des grumes sur les parcs de rupture. A contrario, l'exploitation de plantations forestières permet un accès facile à la ressource, et en grande quantité. Ainsi, le sciage rapide des grumes et le séchage des débits permettent l'utilisation des aubiers comme bois d'œuvre. De la sorte, une filière mobilier ou menuiserie intérieure pourrait être alimentée par la production d'aubiers sains et secs. Ces aubiers pourraient également servir en emballage (cagettes) des produits agricoles. Une valorisation optimum de la ressource produite reste un atout important pour la réussite économique d'une filière «bois issus de plantation ».

\section{Conclusion}

Les cinq espèces présentées ont un fort potentiel pour le développement d'une filière industrielle de bois d'œuvre issus de plantation. Les besoins futurs de la Guyane dans ce secteur seront importants. Alimenter le marché de construction local par du bois d'œuvre produit sur place est une bonne opportunité économique et écologique. Fournir une filière de bois de charpente avec des bois de plantation produits selon des itinéraires durables est un défi qu'il faudra relever. Les bois de Cordia alliodora et Tarrietia utilis semblent avoir les meilleurs potentiels technologiques pour alimenter cette filière bois de charpente, bien que les trois autres puissent potentiellement y subvenir aussi. Simarouba amara et Vochysia tomentosa auront davantage de potentiel en menuiseries intérieures et en emballage, alors que Bagassa guianensis sera mieux placé sur le marché de la menuiserie extérieure, place que cette essence occupe actuellement avec les provenances de forêt naturelle. II serait d'ailleurs davantage raisonnable de ne pas se limiter à la sylviculture d'une seule espèce et de profiter du potentiel de création de filière de plusieurs de ces cinq candidates.

\section{Remerciements}

Ce travail a été conduit dans la cadre du projet ForesTreeCulture (Étude du développement et des propriétés du bois de plantation d'espèces forestières guyanaises) et a bénéficié du soutien financier du Fonds européen de développement régional (FEDER, accord $n^{\circ} 31703$ ). Nous tenons à remercier Onoefé Ngwete et Soepe Koese pour nous avoir aidés dans le travail de terrain et de découpe en atelier.

\section{Références bibliographiques}

Baillères H., Calchera G., Demay L., Vernay M., 1998. Classement mécanique des bois guyanais de structure selon trois techniques non destructives. Bois et Forêts des Tropiques, 257 : 47-62. http://bft.cirad.fr/cd/BFT 257 47-62.pdf

Bouillet J.-P., Hueber R., 1985. Note sur un essai sylvicole sur l'Okoumé (Aucoumea klaineana). Station de Paracou. Nogent-sur-Marne, France, Cirad-CTFT, 43 p. http://agritrop. cirad.fr/361936/

Bouillet J.-P., Sutter E., Hueber R., 1985. Note sur les introductions d'espèces exotiques. Mise en place en 1984. Stations de Paracou et de Combi, Guyane. Kourou, France, Cirad-CTFT, 77 p. http://agritrop.cirad.fr/361992/

De Chatelperron G., Hueber R., 1987. Préservation des essais mis en place en 1986 sur les stations de Paracou et de Combi. Rapport CTFT Guyane, Division Sylviculture, 40 p. http://infodoc.agroparistech.fr/index.php?lvl=notice display\&id=141332 
Clair B., Thibaut B., 2014. Physical and mechanical properties of reaction wood. In: Gardiner B., Barnett J., Saranpää P., Gril J. (Eds). The biology of reaction wood. Springer Series in Wood Science, 171-200.

Desalegn G., 2012. Durability of Cordia alliodora timber and effectiveness of preservatives in protecting timber against termites and fungal attacks. In: Tadesse W., Desalegn G., Yirgu A. (Eds). Forestry and Forest Products: technologies and issues. Addis Ababa, Ethiopia, Ethiopian Institute of Agricultural Research, 361-374.

Dupuy B., Chézeaux E., 1994. La sylviculture du Niangon en plantation. Bois et Forêts des Tropiques, 239 : 9-22. http:// bft.cirad.fr/cd/BFT 239 9-22.pdf

Gérard J., Guibal D., Cerre J.-C., Paradis S., 2016. Atlas des bois tropicaux. Versailles, France, Quæ, 1000 p.

Ghislain B., Clair B., 2017. Diversity in the organisation and lignification of tension wood fibre walls - a review. IAWA Journal, 38 (2): 245-265. https://doi.org/10.1163/2294193220170170

Guitet S., 2005. Mieux valoriser la ressource en bois et réduire les impacts sur l'environnement : deux objectifs convergents pour une exploitation durable en Guyane française. Rendez-Vous Techniques de l'ONF, 9 : 65-69. http://www.onf.fr/++lang++en/outils/medias/20080707090008-8855/++files++/2

Hueber R., Mauranges P., 1984a. Note sur les essais mis en place en 1983 et 1984. Stations de Paracou et de Combi. Nogent-sur-Marne, France, Gerdat-CTFT, 82 p. http://agritrop.cirad.fr/361812/

Hueber R., Mauranges P., 1984b. Note sur diverses introductions à la station de Paracou. Rapport CTFT Guyane, Division Sylviculture, $65 \mathrm{p}$.

Hueber R., 1992. Note sur la mise en place de parcelles conservatoires d'Acacia mangium et crassicarpa. Kourou, France, Cirad-Forêt, 80 p. http://agritrop.cirad.fr/333023/

Longwood F. R., 1962. Present and potential commercial timbers of the Caribbean: with special reference to the West Indies, the Guianas, and British Honduras. Washington, DC, USA, United States Department of Agriculture, Forest Service, $167 \mathrm{p}$.

Marien J.-N., Mallet B., 2004. Nouvelles perspectives pour les plantations forestières en Afrique centrale. Bois et Forêts des Tropiques, 282 : 67-79. http://bft.cirad.fr/cd/ BFT 282 67-79.pdf

Meier E., 2015. Wood! identifying and using hundreds of woods worldwide. The Wood Database, $272 \mathrm{p}$.

NF EN 350, 2016. Durabilité du bois et des matériaux dérivés du bois - Méthodes d'essai et de classification de la durabilité vis-à-vis des agents biologiques du bois et des matériaux dérivés du bois. Directive européenne.

Nicolini E., Morel H., 2016. « ForesTreeCulture ». Étude du développement et des propriétés du bois de plantation d'espèces forestières guyanaises. Cirad-ONF, PO FEDER 2007-2013, projet $n^{\circ} 31707,28$ p. https://antilles-guyane. cirad.fr/content/download/6261/45224/version/1/file/ ForesTreeCulture.pdf
Normand D., 1966. Les Koualis, Vochysiacées de Guyane, et leurs bois (première partie). Bois et Forêts des Tropiques, 110 : 3-11. http://bft.cirad.fr/cd/BFT 110 3-11.pdf

Paradis S., Guibal D., Vernay M., Beauchêne J., Brancheriau L., Cabantous B., 2011. Tropix 7. Caractéristiques technologiques de 245 essences tropicales et tempérées. Montpellier, France, Cirad. Nouvelle version de Tropix 6.0, 2008. https://doi.org/10.18167/74726F706978

Pirard R., Dal Secco L., Warman R., 2016. Do timber plantations contribute to forest conservation? Environmental Science \& Policy, 57: 122-130. https://doi.org/10.1016/j. envsci.2015.12.010

R Development Core Team, 2011. R: A Language and Environment for Statistical Computing. Vienna, Austria, R Foundation for Statistical Computing. http://www.R-project.org/

Schimann H., Bach C., Lengelle J., Louisanna E., Barantal S., Murat C., Buée M., 2017. Diversity and structure of fungal communities in neotropical rainforest soils: the effect of host recurrence. Microbial Ecology, 73: 310-320. https:// doi.org/10.1007/s00248-016-0839-0

Sutter E., Bouillet J.-P., 1985. Essais de comportement d'essences locales guyanaises. Station de Paracou. Situation au 30.11.1985. Kourou, France, Cirad-CTFT, 100 p. http://agritrop.cirad.fr/361986/

XP ENV 807, 2001. Produits de préservation du bois - Détermination de l'efficacité vis-à-vis des micro-organismes de pourriture molle et d'autres micro-organismes du sol. Directive européenne. https://www.boutique.afnor.org/norme/ v2/xp-env-807/produits-de-preservation-du-bois-determination-de-l-efficacite-vis-a-vis-des-micro-organismes-depourriture-molle-et-d-autres-/article/663597/fa101930?x-

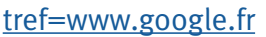

XP CEN/TS 15083-1, 2006. Durabilité du bois et des matériaux dérivés du bois - Détermination de la durabilité naturelle du bois massif vis-à-vis des champignons lignivores - Méthodes d'essai - Partie 1 : Basidiomycètes. Directive européenne. https://www.boutique.afnor.org/norme/v2/ xp-cen-ts-15083-1/durabilite-du-bois-et-des-materiaux-derives-du-bois-determination-de-la-durabilite-naturelle-dubois-massif-vis-a-vis-des-cham/article/660070/fa112133?xtref $=$ www.google.fr

XP CEN/TS 15083-2, 2006. Durabilité du bois et des matériaux dérivés du bois - Détermination de la durabilité naturelle du bois massif vis-à-vis des champignons lignivores - Méthodes d'essai - Partie 2 : Micro-organismes de pourriture molle. Directive européenne. https://www.boutique. afnor.org/norme/v2/xp-cen-ts-15083-2/durabilite-dubois-et-des-materiaux-derives-du-bois-determination-dela-durabilite-naturelle-du-bois-massif-vis-a-vis-des-cham/ article/697421/fa112134?xtref=www.google.fr 


\section{Annexe 1.}

Profils moyens radiaux et longitudinaux (à gauche) et diagramme des proportions de matière à $1 \mathrm{~m} 30$ (à droite) chez les cinq espèces étudiées. La ligne en pointillé passant par zéro symbolise la moelle alors que les autres lignes symbolisent la limite aubier/duramen (d'après le Script $R$ de Romain Lehnebach).
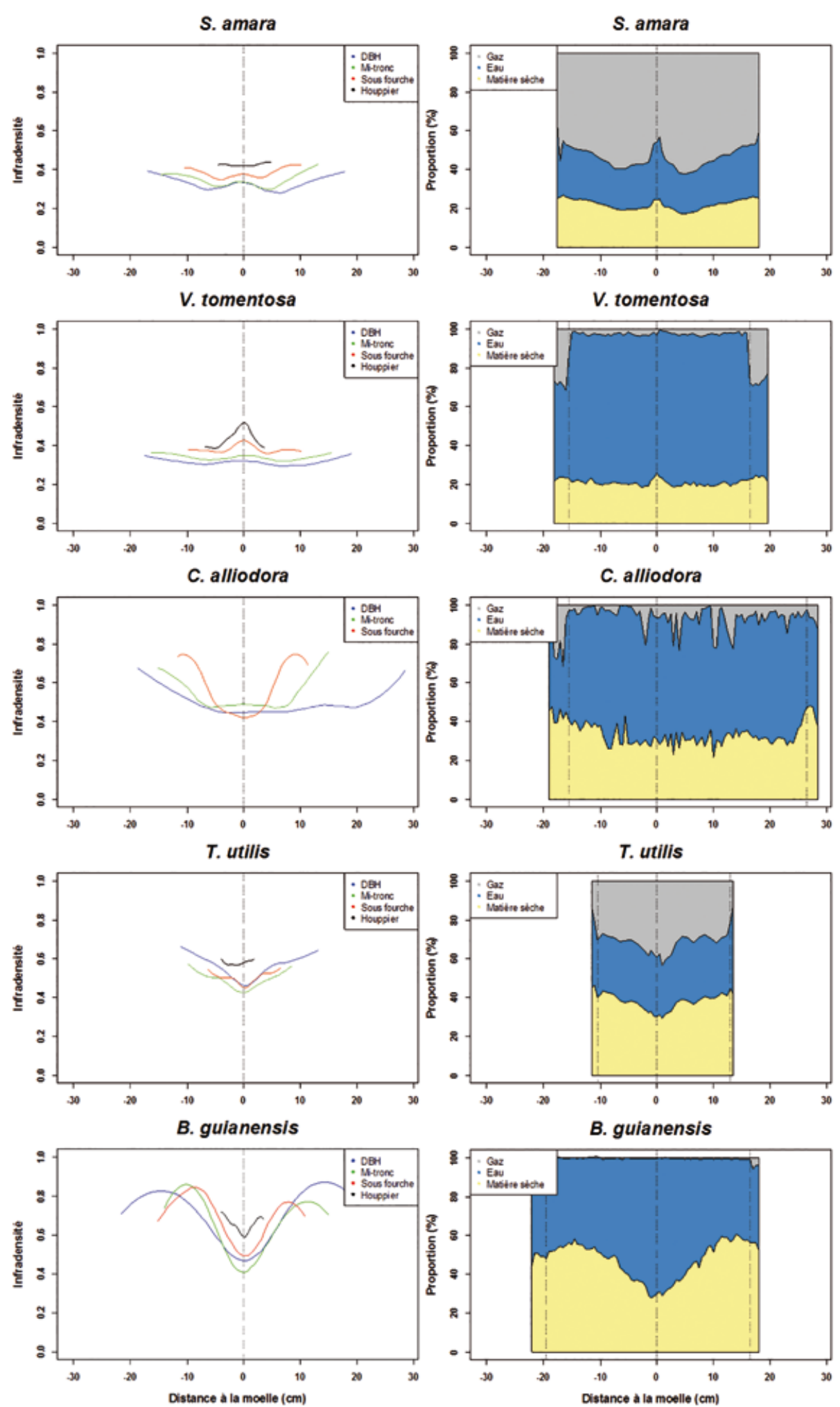
74 BOIS ET FORÊTS DES TROPIQUES, 2017, N³34 (4)

4 FOCUS / PLANTATION TIMBER IN FRENCH GUIANA

Annexe 2.

Comparaison des résultats des principales propriétés étudiées avec les valeurs de référence sur les arbres de forêt naturelle. Les traits en bleu représentent les plages de variation des valeurs mesurées entre le premier et le troisième quartile (75\% des mesures). Les croix en rouge correspondent aux valeurs moyennes de l'Atlas des bois tropicaux (Gérard et al., 2016). Pour la perte de masse, les classes de durabilité ont été reconverties en perte de masse en se référant à la norme NF EN 350.

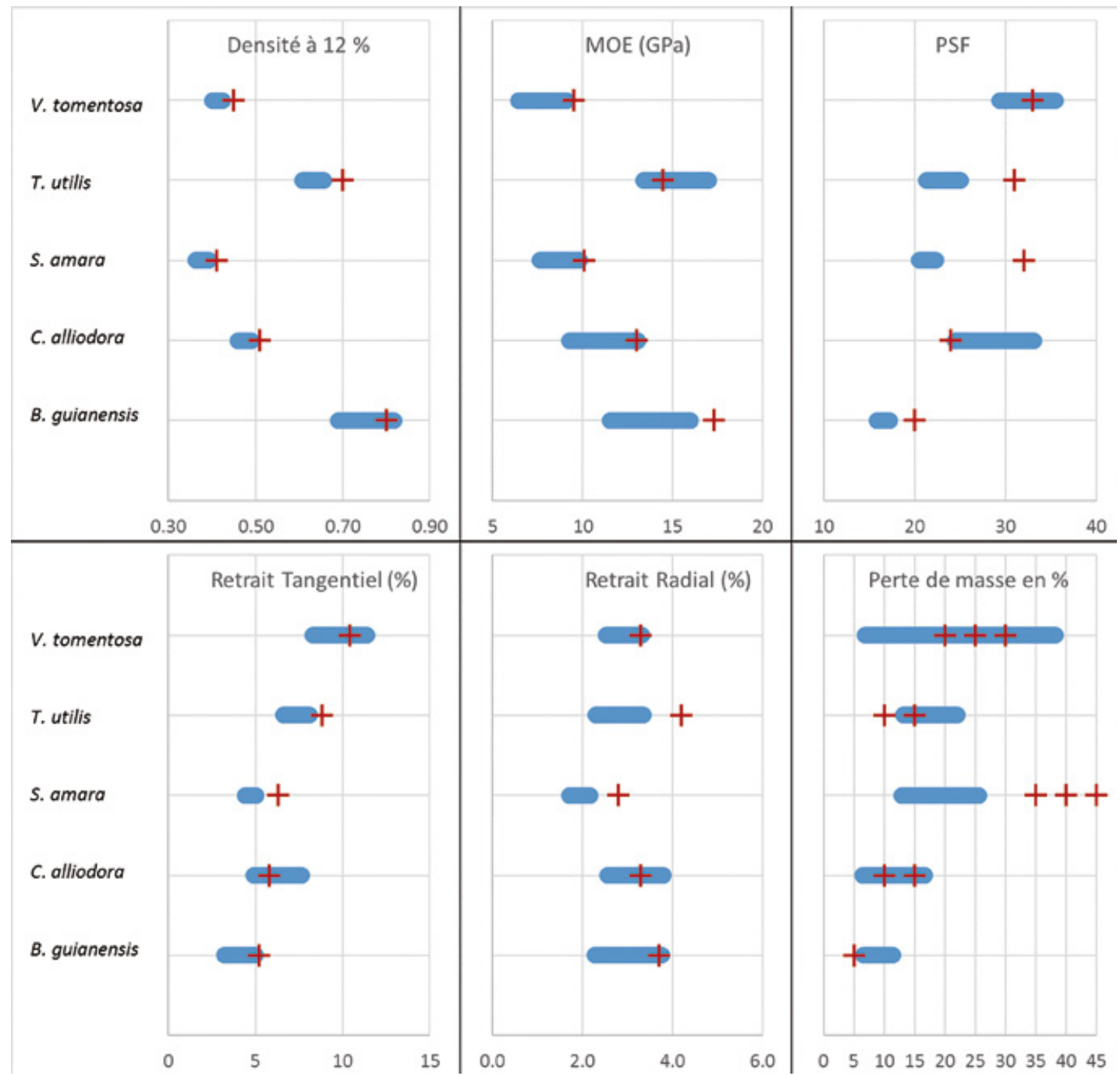

